\title{
Periphere Stammzellen oder Knochenmark transplantieren?
}

\author{
Bei Verwendung von Stammzellen aus dem peripheren Blut \\ HLA-identischer Spender wurden ebenso gute Ergebnisse wie \\ nach einer Knochenmarktransplantation beschrieben. Anasetti \\ et al. haben nun in einer randomisierten Multizenterstudie \\ untersucht, ob dies auch für nicht verwandte Spender gilt. \\ N Engl J Med 2012; 367: 1487-1496
}

551 Patienten unter 66 Jahren mit verschiedenen Formen der Leukämie wurden zwischen 2004 und 2009 an 48 Zentren in den USA und Kanada transplantiert. Den Spendern wurden randomisiert entweder Stammzellen nach Stimulation aus dem peripheren Blut oder Knochenmark entnommen. Die mittlere Nachbeobachtungsdauer betrug 36 Monate. Primärer Endpunkt war das 2-Jahres-Überleben, sekundäre Endpunkte unter anderem Transplantatversagen, Graft-versusHost-Disease (GvHD), Rezidive und Infektionen.

Den primären Endpunkt erreichten in der Stammzellgruppe $51 \%$, in der Knochenmark-Gruppe 46\% ( $p=0,29)$. Auch das krankheitsfreie Überleben nach 2 Jahren und die Rezidivrate unterschieden sich nicht. Bei der Rate unerwünschter Ereignisse gab es ebenfalls keinen signifikanten Unterschied. Stammzelltransplantate nahmen signifikant schneller die Funktion auf, Transplantatversagen ereignete sich in der Stammzell-Gruppe signifikant seltener. Eine akute GvHD trat in beiden Gruppen gleich häufig auf, eine chroni- sche GvHD in der Knochenmark-Gruppe signifikant seltener. Eine immunsuppressive Therapie war bei den Stammzellpatienten mit 57 vs. 37\% nach 2 Jahren häufiger nötig $(\mathrm{p}=0,03)$.

Die Autoren halten eine Transplantation peripherer Stammzellen vor allem bei Patienten mit hohem Risiko eines Transplantatversagens für sinnvoll, eine Knochenmarktransplantation dagegen für alle anderen Patienten, insbesondere bei solchen, die schon durch vorangehende Chemotherapien immunsupprimiert sind.

\section{Fazit}

Die Autoren kommen zu dem Schluss, dass beide Ansätze gleiche Raten für Überleben, Rezidive und akute GvHD aufwiesen. Das Anwachsen des Transplantats sei aber besser bei Patienten nach Stammzelltransplantation, dafür sei die Häufigkeit einer chronischen GvHD nach einer Knochenmarktransplantation geringer.

\section{Dr. Peter Pommer, Oberammergau}

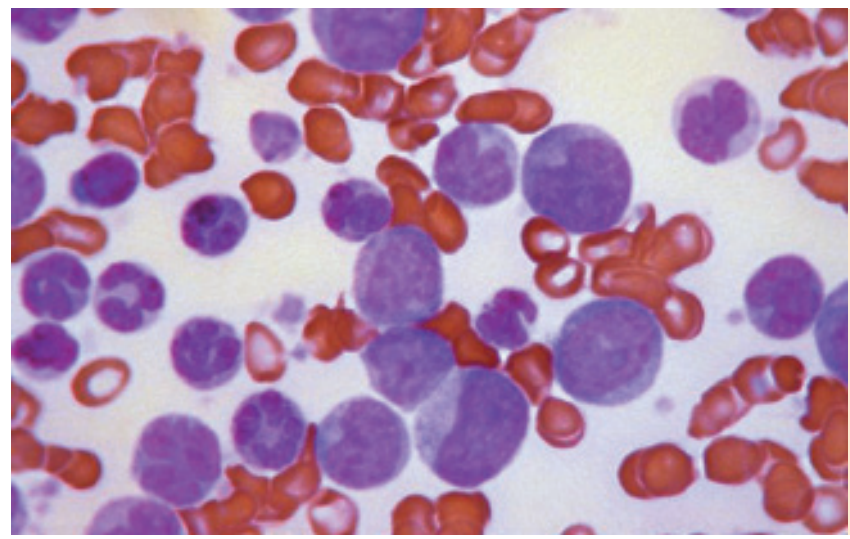

Laut den Autoren wächst das Transplantat bei einer Stammzelltransplantation im Vergleich zur Knochenmarktransplantation besser an, bei letzterer tritt dafür seltener eine chronische GvHD auf. Bild: Blastenkrise bei chronischer myeloischer Leukämie (Quelle: CDC, Fotograf: Stacy Howard). 